\title{
Incentivizing Consumer Sharing in Social Media: The Role of Audience Size: An Abstract
}

\author{
Yueming Zou and Yuping Liu-Thompkins
}

\begin{abstract}
A key issue in social media marketing is insufficient consumer participation and engagement. Most economic exchanges which are used to stimulate social sharing failed to consider the social dynamics of the social media environment. This research aims to answer the following research question: how can companies target different conditions with different incentives to maximize consumer sharing through social media? The experimental study was conducted. The findings of the study show that non-monetary incentives are more effective when sharing to a wide audience is requested, but incentive type does not make a difference when sharing is limited to specific individuals.
\end{abstract}

Reference Available Upon Request

\footnotetext{
Y. Zou $(\bowtie)$

Longwood University, Farmville, VA, USA

e-mail: zouy@longwood.edu

Y. Liu-Thompkins

Old Dominion University, Norfolk, VA, USA

e-mail: yxxliu@odu.edu
} 